\title{
The Heart failure treatment, All at once!
}

\author{
Jiajun Shen ${ }^{1}$ \\ ${ }^{1}$ Haiyuan College, Kunming Medical University, Yunnan, China \\ $+8615687854207$
}

\begin{abstract}
Heart failure (HF) shows great public health concerns and it is important to cure the disease. For now nearly 20 million people are influenced and the number of the effected people is still arising. HF is a complicated disease which can be induced from lots of reasons, including disruption of myocardial function, obesity and diabetes. According to the clinical guidance, the types of HF are different due to different standards, which means the treatments are also different as well. Here we focused on the mainly treatment of HF like drug treatment and non-drug treatment, listing the key performance of each therapy. At last, we summarized the whole picture of the treatment of $\mathrm{HF}$ and gave several directions of the future development of treatments on HF.
\end{abstract}

\section{Heart failure}

Heart failure (HF) is a public health disease, affecting almost 20 million people worldwide, with a projected $25 \%$ increase in morbidity by the next ten years. Gradually, $\mathrm{HF}$-associated health care outgoings are going to more than two times by 2030.For example, present US costs for HF have been forecasted for $\$ 30$ billion. What's more, HF deeply influences the lives of those suffering from the disease, leading to serious morbidity and mortality [1].

$\mathrm{HF}$ is an intricate clinical syndrome that can be due to exceptional situation myocardial function (systolic and diastolic function), valvular or precardiac illness, where heart shows an enlarged volume (Figure 1). Either of these causes impairs forward flow of blood with barrier fluid retention, always accompanying pulmonary congestion, peripheral edema, breathing difficulties and wired [2].

The HF references from both sides of the Atlantic advice distinguish between HF with decreasing ejection fraction (HFrEF) and HF with saved ejection fraction (HFpEF). Each comprises almost half of the HF total population. HFrEF and HFpEF encompass diverse demographics and are associated with different proportions of morbidities and reactions to medical interventions. As a matter of fact,though the accurate offset for keeping the ejection fraction (EF) is controversial (EF ranges from $40 \%$ to $55 \%$ ), the presence of HFrEF hints a different method in responding to current medical HF treatment when compared with $\mathrm{HFpEF}$, regardless of the precise EF cut-point for HFpEF used. Furthermore, the most of therapeutic method approaches for HF, including both medical and facility therapy, have specific targeting or show benefits in the HFrEF population compared with HFPEF population [3$5]$.

1901110384@pku.edu.cn, Shenjiajun1116@163.com

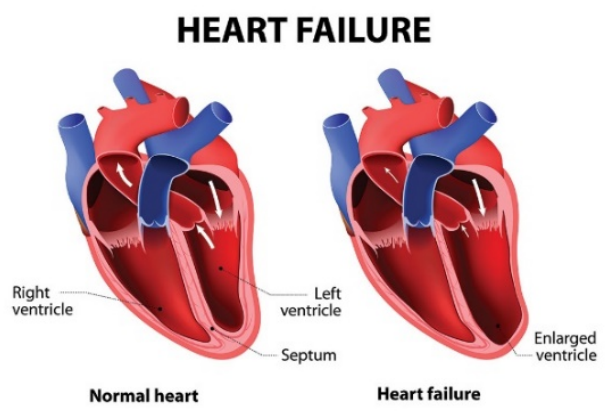

Fig1. Normal heart and heart failure

\section{Reasons of HF}

Heart failure (HF) is caused by a variety of reasons. Classifications of HF have proved to be exceedingly complex in many respects. HF frequently develops from intrinsic myocardial texture or functional abnormalities. This dysfunction was firstly called cardiomyopathy in 1957. In clinical, the initial difference in categorizing a cardiomyopathy, expressly in the setting of HFrEF, is ensuring whether the myocardial dysfunction is related to coronary artery diseases(CAD).CAD is always assessed by either invasive or noninvasive manners $[2,7,8]$.

The remedy of ischemic cardiomyopathy (ICM) with revascularization may ameliorate or settle the myocardial dysfunction. Then, the identification of an potential systemic condition or genetic disease may allow for disease-specific therapy and may also stick out other interrelated sequelae for which the patient (or kinsfolk) may be at risk [9, 10]. Put forward by Maron and colleagues nonischemic cardiomyopathies (NICM) are segmented into genetic, mixed (some shapes may be genetic in origin) and acquired reasons. In the mixed category, there is a certain proportion $(20 \%-35 \%)$ of those diagnosed as dilated cardiomyopathy (DCM) who 
may have familial cardiomyopathy, interpreted that two or more intimate related family members with DCM [10, 11]. In addition, there are various factors, many of which that are genetically resolved, that can predispose towards to the evolution of a secondary NICM [6].

A mass of progress has lately been made in the discovery and comprehending of the genetics of familial dilated cardiomyopathy (FDC). A consensus has emerged that with the newest of diagnosis of idiopathic dilated cardiomyopathy (IDC), the clinical examination of firstdegree family members will display FDC in at least $20 \%$ to $35 \%$ of those members of family. Point mutations in 31 autosomal and $2 \mathrm{X}$-linked genes on behalf of diverse gene ontogeny have been implicated in generating FDC whereas account for merely $30 \%$ to $35 \%$ of genetic reasons. At present, 33 genes (31 autosomal and $2 \mathrm{X}-$ linked) are identified in non-syndromic DCM. Nextgeneration sequencing ways have memorably reduced sequencing costs, making clinical genetic testing feasible for comprehensive panels of dilated cardiomyopathy genes [10].

There are several treatments for HF, but the tendency of chemotherapy-induced cardiomyopathy (CHIC) has enhancive exponentially over the last years with the augment in chemotherapeutic choices and improved longterm survival rates of patients received with cardiotoxic chemotherapy and radiation[11, 12].In addition to the vital and plentiful cardiovascular (CV) influence of chemotherapy and radiation exposure, including premature $\mathrm{CAD}$ and peripheral vascular illness, diabetes and metabolic syndrome, hypertension, valvular sickness, conduction disease, pericardial disease, and restrictive cardiomyopathy, several kinds of chemotherapeutic agents have been implicated in the process of CHIC[12].

The exponential augment in the morbidity of metabolic syndrome, particularly the obesity and diabetes mellitus (DM) epidemic diseases, has influenced and shaped the track of HF development and evolution over the last 2 decades. Both obesity and DM are autocephalous risk elements for the future progress of HF, with the latter will bring out a worse consequence in those with established HF [2, 13-15].

In terms of obesity, it is an increasingly universal situation that has something to do with augmented cardiovascular risk, including added risk of developing HF. On account of the associations of obesity with cardiac textural and hemodynamic alterations, as well as case reports of backspin of cardiomyopathy with loss of the weight, obesity has been speculated to have a noxious influence in patients with HF. Nevertheless, several recent studies have indicated that in patients with established HF, obesity has nothing to do with increased mortality, but rather is related to improved survival rate. Potential mechanisms of heart protection in obesity include an attenuated activation of the neurohumoral system, strengthen the protection against endotoxin/inflammatory cytokines to enhance nutrition and metabolic reserve.[16].

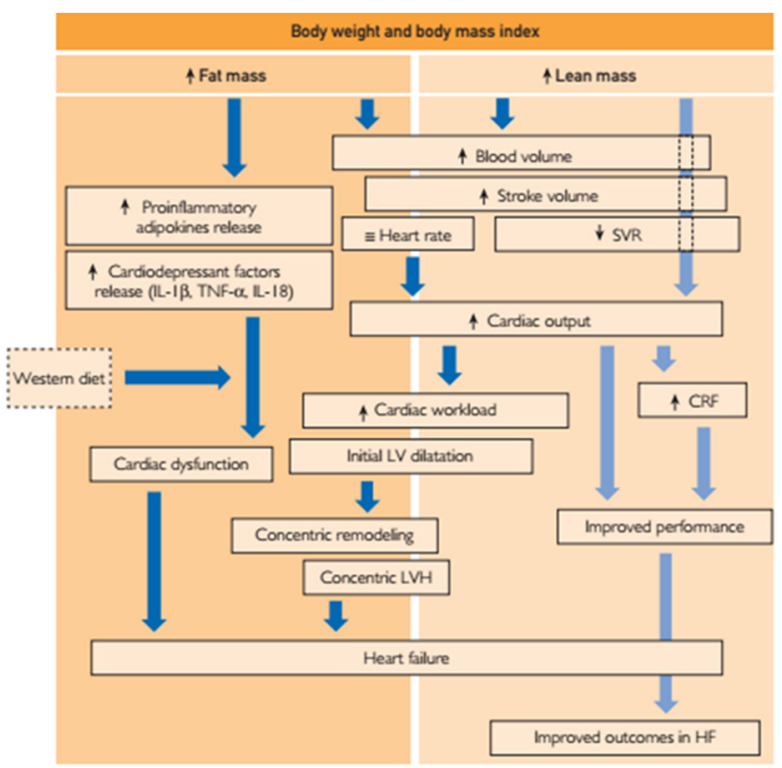

Fig2. Proposed mechanisms driving obesity to heart failure (HF) [17].

Diabetic cardiomyopathy develops via various mechanisms, including augmented oxidative stress, endothelial dysfunction, expedited atherosclerosis, and advanced glycation end-products resulting in enhanced myocardial stiffness and cellulate [18-21].

\section{Drug treatment of HF}

There are lot of treatment to cure HF, including drug treatment and non-drug treatment, and we analyzed the two respects and summarized the situation for now.

\subsection{Hydralazine and Isosorbide Dinitrate:}

A great number of African Americans are impacted by $\mathrm{HF}$, with enhancive morbidity and mortality. In the African-American HF experiment employ BiDil, a medicine uniting isosorbide dinitrate and hydralazine hydrochloride ( The combination are made up of $20 \mathrm{mg}$ of isosorbide and $37.5 \mathrm{mg}$ hydralazine hydrochloride ) dose reveled a $43 \%$ reduce in total mortality when in comparison with placebo and a 39\% lessen in first hospitalization. This fixed dose has some functions that as a nitric oxide enhancer and an antioxidant, as well as aids to prevent tolerance to the prolonged employ of nitrate [22]. The African American Heart Failure Trail (A-HeFT) tested the use of BiDil augmented to standard HF treatment in blacks with New York Heart Institute functional class III and IV HF. At present, BiDil is deemed to the therapy of HF as an auxiliary to standard treatment in black patients [23].

\subsection{Ivabradine}

Higher heart rate (HR) is independently connected with worse endings in multiple cardiac diseases, including hypertension, coronary artery illness, and HF.HR is decided by the pacemaker movement of cells within the sinoatrial node [24]. Ivabradine is a blocker of an I 
diverting electronic current (If) aisle and brings about rate-dependent suppression of the pacemaker movement in the sinoatrial node [25]. Ivabradine is a new fashioned and only practicable $\mathrm{HCN}$ inhibitor. In the CONSTATHE-DHF research, ivabradine decreased HR and ameliorated left ventricular (LV) ejection score, LV diastolic functions, and right ventricle function in brachycranic decompensated HF (ADHF) [26]. And Ivabradine has been authorized for jarless angina and chronic HF in a lot of countries except Japan. According to the SHIFT trial consequences illustrated that ivabradine improves chronic HFrEF prognosis. In a word, for stable $\mathrm{HFrEF}$ patients with $\mathrm{EF}<35 \%$ and $\mathrm{HR}$ greater than 70 $\mathrm{bpm}$, ivabradine improves the prognosis and might be a first selection of treatment, if beta-adrenoceptor antagonists are not endured [25].

\subsection{Aldosterone antagonists}

Aldosterone antagonists are significant pharmacologic cures in the neurohormonal blockade indispensable in the therapy of systolic HF. These medicines have been shown to reduce mortality and lessen hospital readmission speeds. The dominating complication of aldosterone antagonists is hyperkalemia, which can be averted to opportune patient choice and earnest monitoring [27]. By combining aldosterone with Mineralcoticosteroid Receptors MR, activating them as intracellular transcription elements. Furthermore, aldosterone, through its functions on $\mathrm{MR}$, and on another not absolutely explored class of receptors, triggers a few signaling pathways that produce sudden, non-genomic functions. In the vasculature, all these variations make for setting up inflammation and cardiovascular dysfunction which lead to or aggravate various cardiovascular $d$ illnesses. But Aldosterone has some merits, it can through effect on MR,commands body fluid and electrolyte balance[28]. And according to references recommend to think about aldosterone antagonists in patients with a serum creatinine less than $2.5 \mathrm{mg} / \mathrm{dL}$ (or an estimated glomerular filtration rate $>30 \mathrm{~mL} / \mathrm{min} / 1.73 \mathrm{~m} 2$ ), without nearest worsening and serum potassium is inferior to $5.0 \mathrm{mEq} / \mathrm{L}$ without a history of serious Hyperkalemia [2].

\subsection{ACEI and ARB}

Although conventional renin-angiotensin system antagonists containing angiotensin-converting enzyme inhibitors and angiotensin receptor blockers have completely changed the remedy of cardiovascular disease (CVD), the crucial PARADIGM-HF treatment exemplary that sacubitril/valsartan, an angiotensin receptorneprilysin inhibitor (ARNI), was predominant to an angiotensin-converting enzyme inhibitor in decreasing cardiovascular morbidity and mortality in sick persons with HF and lessened ejection fraction[29]. Another studies conducted to inspect the curative effect of Angiotensin II Receptor Blockers (ARB), including irbesartan and candesartan, Angiotensin-Converting Enzyme Inhibitors (ACEI), containing perindopril, in the remedy of $\mathrm{HFpEF}$, revealed moderate virtue but no obvious advantages[30]. The results of the experiment showed comparison with the control group, patients treated with ACEI had a 25 percent reduction in mortality[31]. ARB is not advantageous to ACEI in increasing mortality and a backup method for patients who unendurable to ACEI[32].

In short, each drug has its own unique method of application. They can interact either individually or interactively.

\section{Non-drug treatment}

\subsection{CRT}

Since the first report showed about biventricular pacing in 1994, cardiac resynchronization therapy (CRT) is an effective therapy selection for advanced $\mathrm{HF}$ and ventricular conduction delay. CRT improves myocardial effected by resynchronizing myocardial contraction, which leads to reverse left ventricular reconstitution. By various clinical experiments have shown that CRT improves cardiac function, as well as monolithic quality of livelihood, and decreases $\mathrm{HF}$ hospitalizations, health care expenses, and mortality. In spite of the stepped-up progress of CRT device technique and its enhancive application to HF patients' treatment, nearly one-third of these patients have non-respond to the remedy or gain some clinical advantages from device implantation.CRT remaining a primary challenge that faces electrophysiologists and research workers [33, 34].

\section{$4.2 \mathrm{CCM}$}

Cardiac contractility modulation (CCM) signs, consisting of bidirectional high-handed bipolar signs transmitted to the right ventricular septum during the categorical refractory period, have been proved to improve conditions, motion tolerance and mass of livelihood and decrease the ratio of HF hospitalizations in patients with EF between $25 \%$ and $45 \%$. CCM treatment is currently permitted to the European Union, China, India, Australia and Brazil for employ in symptomatic HFrEF patients with normal or mildly extended QRS continuance. CCM is expressly salutary in patients with baseline EF between $35 \%$ and $45 \%$, which contains half the scope of HF patients with mid-range $\mathrm{EF}(\mathrm{HFmrEF})$. At the cellulate standard, CCM has been proved to HFrEF patients to become better in calcium handling, to reverse the fetal myocyte gene project related to $\mathrm{HF}$, and to accelerate reverse reconstitution. $\mathrm{CCM}$ with reserved $\mathrm{EF}$ has the latent capacity to remedy HF, and CCM is believed may fill a significant unmet need in all EF approaches to treating $\mathrm{HF}$ [35].

\subsection{ICD}

Although there are numerous ways to treat $\mathrm{HF}$ at present, the risk of sudden cardiac death (SCD) in HF sustains high. Prophylactic implantable cardioverter defibrillator (ICD) support has been proved to decrease the risk of SCD in 
patients with $\mathrm{HF}$, as well as damaged left ventricular function. In patients with ventricular arrhythmias, a prophylactic ICD will remedy continuous ventricular tachycardia or ventricular fibrillation. Especially as a prophylactic treatment for patients with conspicuous symptomatic HF if their left ventricular ejection fraction is $35 \%$ or less, ICD can be used as a preventive means of therapy. Preventive ICD implantation seems to be a reasonable procedure to decrease mortality by reducing SCD in patients with HF. Nevertheless, ICD implantation is a traumatic operation, and both short- and long-term complications can happen. As a result, we must assess whether the patient is hoped to subsist at least 1 year with favorable functional condition before implanting ICD $[4,5]$. Most important of all the possibility of SCD should over the potential incidents related to therapy method. The application of reformative imaging technique, as well as electrophysiological test of ventricular arrhythmia matrix can ameliorate choice to ICD patients [36].

\section{Summary and future development}

$\mathrm{HF}$ is an intricate clinical syndrome leading to structural or functional obstacles of ventricular engorged or ejection of blood. HF has an undesirable prognosis and the burden to society remains enormous.

So far, many treatments have been explored around the world to deal with HF [37]. According to the classification of drug treatment, ACEI, ARB, Hydralazine, Isosorbide Dinitrate, Aldosterone antagonists and ivabradine have appeared in drug remedy, while CRT, CCM and ICD have appeared in non-drug treatment. Every medicine has its advantages and disadvantages. For example, although aldosterone antagonists have been proved to reduce mortality and hospitalization rate, there are still complications, which may lead to hyperkalemia and aggravation of cardiovascular disease .At present, $\mathrm{CCM}$ can improve symptoms, exercise tolerance, quality of life and reduce the ejection fraction of HF patients between $25 \%$ and $45 \%$, which contains half the scope of HF patients with mid-range EF (HFmrEF). At the cellulite standard, CCM has been proved to HFrE patients to become better in calcium handling, to reverse the fetal myocyte gene project related to HF, and to accelerate reverse reconstitution. CCM with reserved EF has the latent capacity to remedy $\mathrm{HF}$, and CCM is believed may fill a significant unmet need in all EF approaches to treating HF.

Studying heart failure at multiple biological levels can yield different results. The unimplemented expectation is that enlarging our knowledge of the genetic architecture of HF will assist to fleetly advance the quality of risk evaluation, diagnoses, and therapy.

According to Genome-wide Association Studies (GWAS) from bioinformatics analysis, genes are prioritized, and diversiform model organisms are used to obtain functional insights. The promising genes with certain functions can be used in personalized medicine later. Similarly, mutations targeting specific pathogenic genes are expected to protect offspring from HF.
In addition, there are many new drugs under study, and future directions can also focus on the stem cell therapy on patients as it showed great effect on other diseases. The rhythm control in heart failure patients is another key point in improving the clinical roles of different therapy.

Despite the deeply insight of the HF treatment during the past years, much more work need to be pushed in the future. $\mathrm{HF}$ is a progressive disease with a rise of prevalence, which did tons of costs to people. Future studies are required to improve the performances of patients and the life quality of them.

\section{REFERENCE}

1. Heidenreich, P.A. et al. Forecasting the impact of heart failure in the United States: a policy statement from the American Heart Association. Circ Heart Fail 6, 606-619 (2013).

2. Yancy, C.W. et al. 2013 ACCF/AHA guideline for the management of heart failure: a report of the American College of Cardiology Foundation/ American Heart Association Task Force on Practice Guidelines. J Am Coll Cardiol 62, e147-239 (2013).

3. Owan, T.E. et al. Trends in prevalence and outcome of heart failure with preserved ejection fraction. $\mathrm{N}$ Engl J Med 355, 251-259 (2006).

4. Mentz, R.J. et al. Noncardiac comorbidities in heart failure with reduced versus preserved ejection fraction. J Am Coll Cardiol 64, 2281-2293 (2014).

5. Steinberg, B.A. et al. Trends in patients hospitalized with heart failure and preserved left ventricular ejection fraction: prevalence, therapies, and outcomes. Circulation 126, 65-75 (2012).

6. Maron, B.J. et al. Contemporary definitions and classification of the cardiomyopathies: an American Heart Association Scientific Statement from the Council on Clinical Cardiology, Heart Failure and Transplantation Committee; Quality of Care and Outcomes Research and Functional Genomics and Translational Biology Interdisciplinary Working Groups; and Council on Epidemiology and Prevention. Circulation 113, 1807-1816 (2006).

7. McMurray, J.J. et al. ESC Guidelines for the diagnosis and treatment of acute and chronic heart failure 2012: The Task Force for the Diagnosis and Treatment of Acute and Chronic Heart Failure 2012 of the European Society of Cardiology. Developed in collaboration with the Heart Failure Association (HFA) of the ESC. Eur Heart J 33, 1787-1847 (2012).

8. Soman, P. et al. Etiology and pathophysiology of new-onset heart failure: evaluation by myocardial perfusion imaging. J Nucl Cardiol 16, 82-91 (2009).

9. Rau, C.D., Lusis, A.J. \& Wang, Y. Genetics of common forms of heart failure: challenges and potential solutions. Curr Opin Cardiol 30, 222-227 (2015).

10. Hershberger, R.E. \& Siegfried, J.D. Update 2011: clinical and genetic issues in familial dilated 
cardiomyopathy. J Am Coll Cardiol 57, 1641-1649 (2011).

11. Bovelli, D., Plataniotis, G., Roila, F. \& Group, E.G.W. Cardiotoxicity of chemotherapeutic agents and radiotherapy-related heart disease: ESMO Clinical Practice Guidelines. Ann Oncol 21 Suppl 5, v277-282 (2010).

12. Carver, J.R., Szalda, D. \& Ky, B. Asymptomatic cardiac toxicity in long-term cancer survivors: defining the population and recommendations for surveillance. Semin Oncol 40, 229-238 (2013).

13. Kenchaiah, S. et al. Obesity and the risk of heart failure. N Engl J Med 347, 305-313 (2002).

14. Nichols, G.A., Gullion, C.M., Koro, C.E., Ephross, S.A. \& Brown, J.B. The incidence of congestive heart failure in type 2 diabetes: an update. Diabetes Care 27, 1879-1884 (2004).

15. Shindler, D.M. et al. Diabetes mellitus, a predictor of morbidity and mortality in the Studies of Left Ventricular Dysfunction (SOLVD) Trials and Registry. Am J Cardiol 77, 1017-1020 (1996).

16. Horwich, T.B. \& Fonarow, G.C. The impact of obesity on survival in patients with heart failure. Heart Fail Monit 3, 8-14 (2002).

17. Carbone, S., Lavie, C.J. \& Arena, R. Obesity and Heart Failure: Focus on the Obesity Paradox. Mayo Clin Proc 92, 266-279 (2017).

18. Bojunga, J. et al. Antioxidative treatment prevents activation of death-receptor- and mitochondriondependent apoptosis in the hearts of diabetic rats. Diabetologia 47, 2072-2080 (2004).

19. Williams, S.B. et al. Acute hyperglycemia attenuates endothelium-dependent vasodilation in humans in vivo. Circulation 97, 1695-1701 (1998).

20. Karnik, A.A., Fields, A.V. \& Shannon, R.P. Diabetic cardiomyopathy. Curr Hypertens Rep 9, 467-473 (2007).

21. van Heerebeek, L. et al. Diastolic stiffness of the failing diabetic heart: importance of fibrosis, advanced glycation end products, and myocyte resting tension. Circulation 117, 43-51 (2008).

22. Ferdinand, K.C. Isosorbide dinitrate and hydralazine hydrochloride: a review of efficacy and safety. Expert Rev Cardiovasc Ther 3, 993-1001 (2005).

23. Carmody, M.S. \& Anderson, J.R. BiDil (isosorbide dinitrate and hydralazine): a new fixed-dose combination of two older medications for the treatment of heart failure in black patients. Cardiol Rev 15, 46-53 (2007).

24. Ide, T. et al. Ivabradine for the Treatment of Cardiovascular Diseases. Circ J 83, 252-260 (2019).

25. Thorup, L., Simonsen, U., Grimm, D. \& Hedegaard, E.R. Ivabradine: Current and Future Treatment of Heart Failure. Basic Clin Pharmacol Toxicol 121, 8997 (2017).

26. Lee, W.C. \& Fang, H.Y. Ivabradine for the Treatment of Acute Mitral - Regurgitation - Related
Decompensated Heart Failure. Cardiology 144, 97100 (2019).

27. Miller, S.E. \& Alvarez, R.J., Jr. Aldosterone antagonists in heart failure. J Cardiovasc Nurs 28, E47-54 (2013).

28. Pantelidis, P. et al. The Mechanisms of Actions of Aldosterone and its Antagonists in Cardiovascular Disease. Curr Pharm Des 24, 5491-5499 (2018).

29. Ambrosy, A.P. et al. The role of angiotensin receptorneprilysin inhibitors in cardiovascular diseaseexisting evidence, knowledge gaps, and future directions. Eur J Heart Fail 20, 963-972 (2018).

30. Bramblett, T., Teleb, M., Albaghdadi, A., Agrawal, H. \& Mukherjee, D. Heart Failure with Preserved Ejection Fraction: Entresto a Possible Option. Cardiovasc Hematol Disord Drug Targets 17, 80-85 (2017).

31. Deedwania, P.C. \& Carbajal, E. Evidence-based therapy for heart failure. Med Clin North Am 96, 915-931 (2012).

32. Nochioka, K., Sakata, Y. \& Shimokawa, H. Combination Therapy of Renin Angiotensin System Inhibitors and beta-Blockers in Patients with Heart Failure. Adv Exp Med Biol 1067, 17-30 (2018).

33. Katbeh, A. et al. Cardiac Resynchronization Therapy Optimization: A Comprehensive Approach. Cardiology 142, 116-128 (2019).

34. Naqvi, S.Y., Jawaid, A., Goldenberg, I. \& Kutyifa, V. Non-response to Cardiac Resynchronization Therapy. Curr Heart Fail Rep 15, 315-321 (2018).

35. Tschope, C. et al. Cardiac contractility modulation: mechanisms of action in heart failure with reduced ejection fraction and beyond. Eur J Heart Fail 21, 1422 (2019).

36. Gerson, M.C., Abdallah, M., Muth, J.N. \& Costea, A.I. Will imaging assist in the selection of patients with heart failure for an ICD? JACC Cardiovasc Imaging 3, 101-110 (2010).

37. van der Ende, M.Y., Said, M.A., van Veldhuisen, D.J., Verweij, N. \& van der Harst, P. Genome-wide studies of heart failure and endophenotypes: lessons learned and future directions. Cardiovasc Res 114, 12091225 (2018). 